\title{
Pattern Formation of Charged Particles in an Electric Field
}

\author{
Tina Lin, ${ }^{\dagger}$ Shmuel M. Rubinstein, ${ }^{\ddagger}$ Andriy Korchev, ${ }^{\S}$ and David A. Weitz ${ }^{* \dagger, \dagger}$ \\ ${ }^{\dagger}$ Department of Physics, Harvard University, 17 Oxford St., Cambridge, Massachusetts 02138, United States \\ ${ }^{\ddagger}$ School of Engineering and Applied Sciences, Harvard University, 29 Oxford St., Cambridge, Massachusetts 02138, United States \\ ${ }^{\S}$ Cabot Corporation, 157 Concord Rd., Billerica, Massachusetts 01821, United States
}

\section{Supporting Information}

\begin{abstract}
The application of an electric field to a suspension of charged particles can lead to the formation of patterns due to electrohydrodynamic instabilities which remain poorly understood. We elucidate this behavior by visualizing the dynamics of charged carbon black particles suspended in a nonpolar solvent in response to an electric field. As the particles are transported across a microfluidic channel, an instability occurs in which the initially uniform, rapidly advancing particle front develops fingers. Furthermore, when the direction of the applied field is repeatedly switched, the particles localize into a remarkably well-defined periodic pattern which reflects an interplay between the fingering instability and particle diffusion.
\end{abstract}
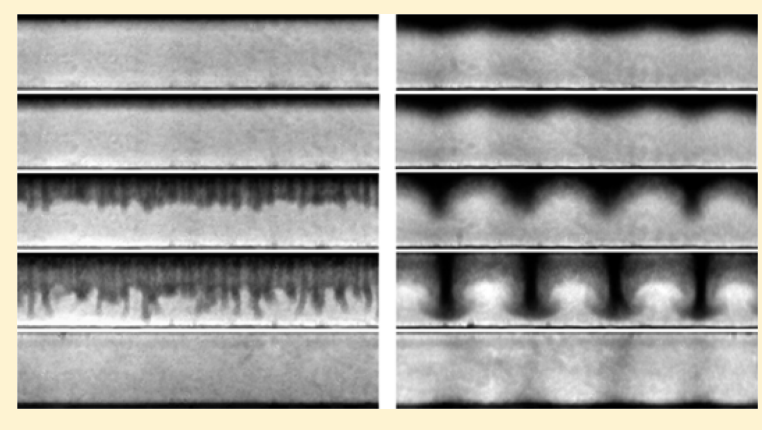

\section{INTRODUCTION}

In nonpolar solvents, charge-induced effects are typically expected to be insignificant due to a high electrostatic barrier to charge dissociation. Nevertheless, charge can be stabilized in nonpolar solvents upon the addition of suitable surfactants; ${ }^{1-4}$ this provides a mechanism to charge and electrostatically stabilize particle dispersions. ${ }^{2-11}$ This effect is utilized in a variety of applications; ${ }^{11,12}$ for example, in electrophoretic displays, the charging of pigment particles in a nonpolar solvent enables them to be transported by an electric field to form an image. ${ }^{13}$ However, achieving the requisite level of control over the trajectories of charged particles is still a major challenge. Upon the application of an electric field, particles in an initially homogeneous dispersion commonly form patterns composed of ordered structures or clusters on the electrode surface; ${ }^{14-17}$ this behavior degrades the performance of electrophoretic displays by limiting image contrast and resolution. Patterns can be modified or even avoided by tuning the strength and frequency of the applied field, the confinement geometry, and the chemical and charge properties of the particle suspension; however, the origin of these patterns remains unclear, making the elimination of their effects and full control over the display difficult. Pattern formation is often attributed to the interplay between electrokinetic and hydrodynamic effects: field-induced electrohydrodynamic flow results in complex particle dynamics, ultimately leading to the aggregation of the particles into pattern-forming structures. ${ }^{18}$ Patterns are visualized after the particles have been deposited on a transparent electrode surface, ${ }^{16,17}$ whereas the particle dynamics are never directly observed. Direct visualization of particle motion through the fluid would help elucidate the relationship between particle dynamics and the formation of patterns. Such knowledge is essential for fully understanding electric-field-induced behavior of charged particles in nonpolar solvents.

Here, we investigate the dynamics and formation of patterns of charged particles in a nonpolar solvent in response to an applied electric field. We use a microfluidic device that allows us to apply an electric field across a suspension of carbon black particles confined within a channel; by combining this device with high-speed imaging, we directly monitor the full motion of the charged particles. Upon being repeatedly driven back and forth across the channel, the particles do form a well-defined pattern. We show that this pattern is due to an instability that occurs when the particles are transported across the channel by a switch in the applied field: as the rapidly advancing particle front is transported across the channel, it breaks into pronounced fingers separated by a well-defined wavelength. This instability is similar to the Rayleigh-Taylor instability for immiscible fluids, but the characteristic wavelength is defined by transverse diffusion of the particles which sets the lower bound on the length scale.

\section{EXPERIMENTAL SECTION}

To investigate charged particle behavior, we use a suspension of $0.5 \mathrm{wt}$ $\%$ carbon black particles dispersed in Isopar G, a nonpolar solvent. To solubilize charge in the solution, $0.5 \mathrm{wt} \%$ surfactant is added as a charge control agent. The surfactant increases the conductivity of the solution to $15000 \mathrm{pS} / \mathrm{m}$; this is more than 4 orders of magnitude higher than the conductivity of pure Isopar G. The carbon black particles have a characteristic size of $140 \mathrm{~nm}$; their surfaces are chemically modified, with polymer brushes that are $3-5 \mathrm{~nm}$, to be positively charged when the charge control agent is added. We fabricate a microfluidic device that allows us to observe the motion of

Received: August 24, 2013

Published: September 17, 2014 
the particles within a microfluidic channel in response to an electric field applied across this channel. The device is fabricated from Norland Optical Adhesive (NOA81) ${ }^{19}$ and consists of three parallel channels: the center channel is $50 \mu \mathrm{m}$ wide, and the two outer channels are 100 $\mu \mathrm{m}$ wide; all of the channels are $25 \mu \mathrm{m}$ high. These channels are separated by a wall that is $10 \mu \mathrm{m}$ wide. The outer channels are filled with a low-temperature solder (Indium Corporation); these form two parallel electrodes through which we can apply an electric field across the center channel. A cross-sectional schematic of the device is shown in Figure 1a. We sonicate the particle suspension to ensure a
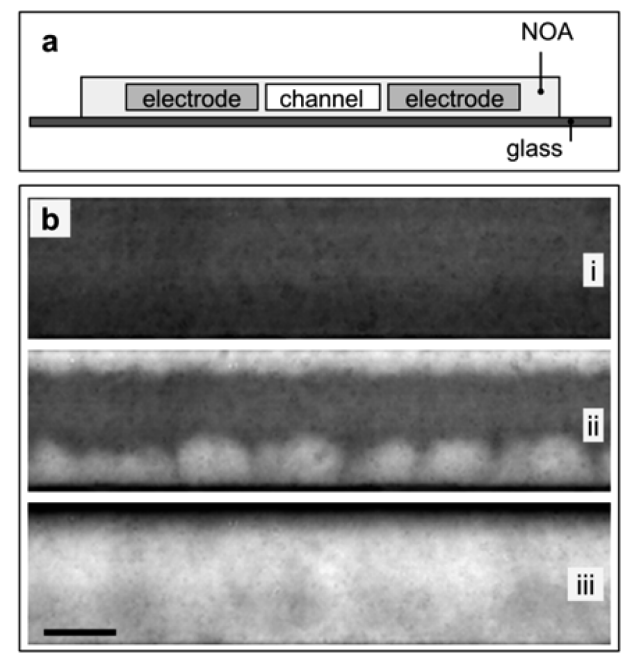

Figure 1. (a) Cross-sectional schematic of microfluidic device. (b) Top view of a channel containing carbon black particles. The particles are initially distributed homogeneously within the channel in the absence of an applied field (i), partially transported to the bottom wall when a field of magnitude $2 \mathrm{~V} / \mu \mathrm{m}$ is first applied across the channel (in the picture, pointing downward) (ii), and completely packed at the top wall when the direction of the field is switched (in the picture, pointing upward) (iii). The particles are transported completely across the channel and packed at the wall after all subsequent switches. Scale bar is $25 \mu \mathrm{m}$.

homogeneous dispersion. The suspension is injected into the center channel of the device. This channel is then sealed with epoxy. The electrodes are connected in series to a function generator (Agilent 33250A) and a high-voltage amplifier (Trek 2210). Particle motion is imaged with a high-speed camera (Phantom V7) at frame rates of up to $10 \mathrm{kHz}$ with a resolution of $800 \times 200$ pixels.

\section{RESULTS AND DISCUSSION}

In the absence of an applied electric field, the particles diffuse freely within the channel and are homogeneously distributed, as shown in Figure 1b,i. When an external field with a magnitude of $2 \mathrm{~V} / \mu \mathrm{m}$ is applied for the first time, the particles are transported toward the negative electrode. Most of the particles are packed against the wall; however, a small subpopulation of particles remains in the central region of the microfluidic channel, as shown in Figure 1b,ii. When the direction of the field is switched, all of the particles are transported completely across the channel and are packed at the opposite wall; this time, in contrast with the initial application of the field, very few particles remain in the bulk, as shown in Figure 1b,iii. This effect likely occurs because a switch in the applied field has a stronger effect on the particles than the initial application of the field: the initial applied field separates charges in the solution; when the applied field is switched, particle motion is driven by both the applied field and the field induced by these separated charges. ${ }^{20}$ Immediately following the switch, the thickness of the particle-rich layer packed against the wall is approximately $l$ $=4 \mu \mathrm{m}$. In all subsequent switches, all the particles are transported across the entire channel and packed at the opposite channel wall.

We periodically alternate the applied field and thereby drive the particles back and forth between the channel walls. The time between consecutive switches, $t_{\mathrm{s}}$, is $1 \mathrm{~s}$. To probe the full details of particle motion, we acquire a sequence of images as the particles are transported across the channel in response to a switch. Initially, the particles are packed homogeneously at the wall. When the field is switched, the particles do not all move simultaneously across the channel; instead of remaining uniform, the particle front develops finger-shaped protrusions that grow longer as the front advances, as shown in the left panel of Figure 2 . Within $50 \mathrm{~ms}$ of the switch, all of the particles

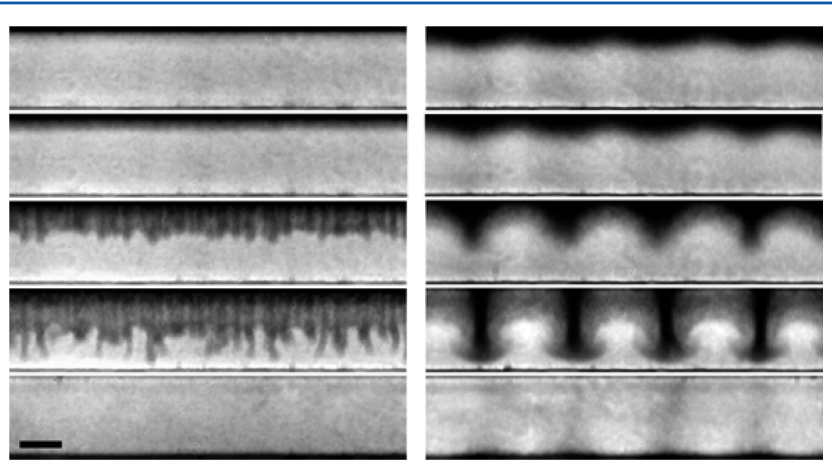

Figure 2. Time series of images of particle transport across the channel in response to a switch in the direction of the applied electric field, for $t_{\mathrm{s}}=1 \mathrm{~s}$ (left) and $t_{\mathrm{s}}=5 \mathrm{~s}$ (right). From top to bottom, the images are at times $0,0.5,1.0,1.5$, and $50 \mathrm{~ms}$ after the switch. Scale bar is $25 \mu \mathrm{m}$.

are packed at the opposite wall. The change in the shape of the particle front from being initially flat to developing fingers during transport occurs for every subsequent switch in the applied field. By contrast, dramatically different behavior occurs when $t_{\mathrm{s}}$ is increased to $5 \mathrm{~s}$. After several switches, the particles are no longer packed homogeneously at the wall following each switch; instead, they become localized in a well-defined, periodic pattern along the wall. The particles in each localized region of higher concentration form plumelike structures as they are transported across the channel, as shown in the right panel of Figure 2; the particles deposit on the opposite wall at locations corresponding to these plumes. Thus, the pattern is maintained through continued switches.

To analyze the fingering instability observed for $t_{\mathrm{s}}=1 \mathrm{~s}$, we use the images to track the time evolution of the particle front as it moves across the channel. By thresholding each image, we determine the profile of the front within the channel at different times, $t$, as shown in Figure 3a. Each of these profiles can be represented by an ensemble of modes with different wavenumbers, $k$. By calculating the fast Fourier transform of each profile, we measure the amplitude of each mode, $A(k, t)$, and determine its temporal evolution. Immediately following the switch, there is little change in $A(k, t)$; however, approximately $0.5 \mathrm{~ms}$ after the switch, $A(k, t)$ grows linearly, as shown in Figure $3 \mathrm{~b}$. Within the time frame of linear growth, we fit the time-dependence of $A(k, t)$ to $A(k, t)=\sigma(k) t$; we thereby obtain a growth rate, $\sigma(k)$, of every mode. We find that $\sigma(k)$ initially increases with increasing $k$, reaching a maximum at $k_{\mathrm{m}} \approx 0.04$ $\mu \mathrm{m}^{-1}$, then decreases for larger $k$. The growth rate curve is 

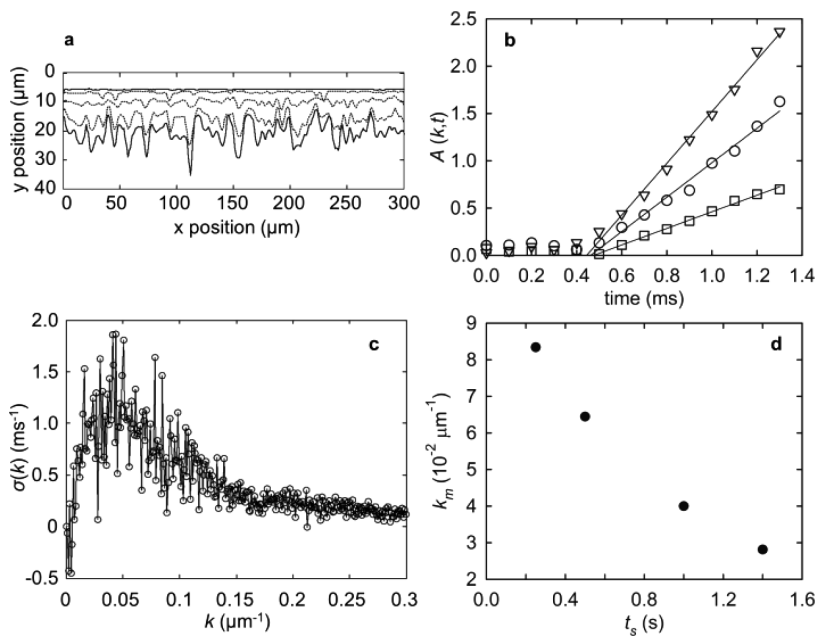

Figure 3. (a) Evolution of the profile of the particle front for a typical example, for $t_{\mathrm{s}}=1 \mathrm{~s}$. The first trace, the nearly flat solid line, is taken at the moment the direction of the field is switched, at $t=0$. The interval between each trace is $0.3 \mathrm{~ms}$. (b) $A(k, t)$ vs time for $k=0.037(\triangle)$, $0.030(\bigcirc)$, and $0.076 \mu \mathrm{m}^{-1}(\square) ; t_{\mathrm{s}}=1 \mathrm{~s}$. The solid lines are linear fits. (c) Growth rate vs wavenumber for $t_{\mathrm{s}}=1 \mathrm{~s}$. (d) Fastest-growing wavenumber decreases with increasing $t_{s}$.

broad, reflecting the rapid growth of a wide range of modes. The fastest-growing mode, $k_{\mathrm{m}}$, corresponds to a fastest-growing wavelength, $\lambda_{\mathrm{m}}$, of $\lambda_{\mathrm{m}}=k_{\mathrm{m}}{ }^{-1}=25 \mu \mathrm{m}$, as shown in Figure 3c. At $k \approx 0.15 \mu \mathrm{m}^{-1}, \sigma(k)$ levels off to a relative minimum. The ratio between this cutoff wavenumber and $k_{\mathrm{m}}$ is approximately 3.8 .

This fingering behavior is reminiscent of the RayleighTaylor instability, which occurs for a system of two layers of fluid, in which the denser fluid is above the less dense fluid. ${ }^{21}$ As gravity drives the heavier fluid downward, the interface between the fluids is unstable and perturbations in the interface grow and develop into fingers, similar to that observed in our system. Within the framework of the classical Rayleigh-Taylor instability, the growth of perturbations is rapid for a wide range of wavenumbers and is reduced at higher wavenumbers because of surface tension: for two immiscible fluids, surface tension suppresses the growth of smaller-wavelength perturbations by minimizing the surface area of the fluid-fluid interface. However, in our system, particles may move freely from the particle-rich region to the particle-free region; thus, the two fluids are miscible and there is no surface tension to serve as the cutoff mechanism. Therefore, there must be another mechanism that causes the large-wavenumber cutoff in our system.

A clue to the cause of the large-wavenumber cutoff comes from the behavior of the particles during the time between switches. After the particles have been transported across the channel and packed at the wall by the constant applied field, they appear to diffuse and the thickness of the particle-rich region grows with time, as shown in Figure 4a; the field remains on during this time. We measure this thickness by measuring the distance between the edge of the particle-rich region and the wall and find that its growth is characteristic of diffusion, exhibiting $t^{1 / 2}$ scaling and behaving as $5.9 t^{1 / 2}$, as shown in Figure 4b; noting that the characteristic length for diffusion in one dimension grows as $(2 D t)^{1 / 2}$, where $D$ is the diffusion constant, this corresponds to $D=17.5 \mu \mathrm{m}^{2} / \mathrm{s}$. For comparison, we use the Stokes-Einstein relation to calculate the single particle diffusion constant for carbon black particles in Isopar
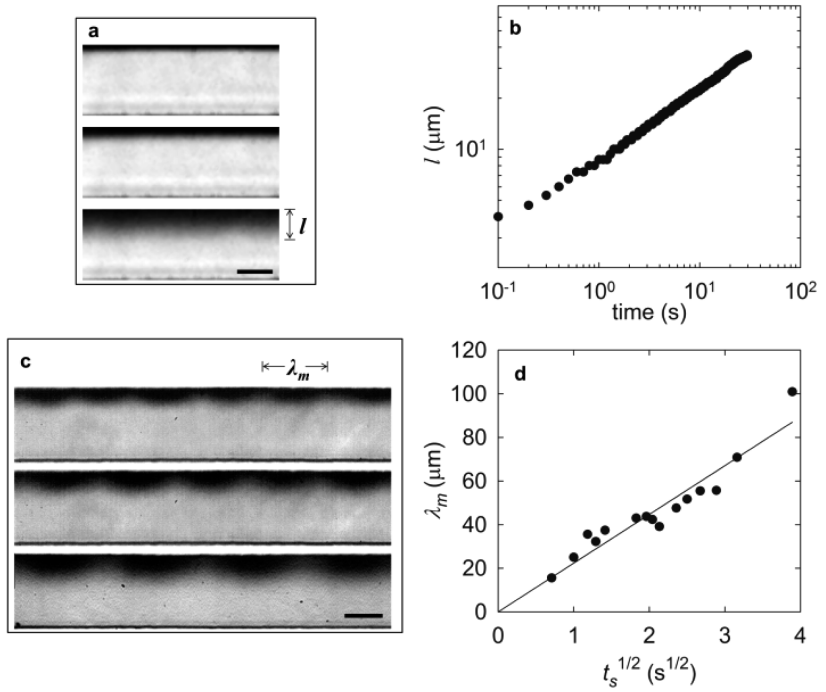

Figure 4. (a) Images of the channel after the particles are packed at the wall by a switch in the applied field at times 0.5 (top), 2 (middle), and $10 \mathrm{~s}$ (bottom) after the switch. Scale bar is $25 \mu \mathrm{m}$. (b) Thickness of particle-rich region vs time; the field is switched at time $=0 .(\mathrm{c})$ Images of the channel after many switches, for $t_{\mathrm{s}}=3.33$ (top), 5 (middle), and $10 \mathrm{~s}$ (bottom). The particles have formed a pattern at the wall. Scale bar is $25 \mu \mathrm{m}$. (d) $\lambda_{\mathrm{m}}$ scales as the square root of time between consecutive switches. The solid line is a linear fit.

and find $D \approx 1.4 \mu \mathrm{m}^{2} / \mathrm{s}$, which is smaller than the measured value. This suggests that the diffusion we observe is not the simple self-diffusion given by the Stokes-Einstein relation, but is instead a collective diffusion due to the repulsive interparticle interactions from the surface charges, which is typically much faster. Additionally, by measuring the progression of the particles at the edge of the particle-rich region, we are tracking the behavior of the fastest particles. The particles not only diffuse normal to the wall, but they also diffuse transverse to the wall. This is the likely cause for the decrease in $\sigma(k)$ at large $k$ : transverse particle diffusion washes out the smaller-wavelength perturbations; therefore the growth of these perturbations is minimized. A longer diffusion time would wash out increasingly larger wavelengths, thus shifting the large- $k$ cutoff, and therefore $k_{\mathrm{m}}$, to smaller values.

To test this idea, we vary the amount of time between switches and analyze the fingering instability during particle transport; we find that increasing $t_{\mathrm{s}}$ indeed corresponds to a decrease in $k_{\mathrm{m}}$, as shown in Figure $3 \mathrm{~d}$. When $t_{\mathrm{s}}>2 \mathrm{~s}$, we observe the formation of a well-defined pattern along the wall during the time between switches; below this critical $t_{\mathrm{s}}$, the particle distribution between switches appears homogeneous. The well-defined pattern consists of a distribution of particles along the wall in which the areas of higher particle concentration are periodically spaced. This effect is due to the nature of particle deposition: the fastest-growing fingers during particle transport reach the wall first, suggesting that particles are deposited in higher amounts at locations corresponding to the fastest-growing fingers. At lower $t_{\mathrm{s}}$, where $\lambda_{\mathrm{m}}$ is smaller, the distance between neighboring fingers is small; therefore, the distance between areas of higher deposition is also small. This distance is small enough such that the overall deposition on the wall is nearly homogeneous. By contrast, at higher $t_{\mathrm{s}}, \lambda_{\mathrm{m}}$ is larger; this results in a more heterogeneous deposition of particles. Thus, for the next switch, the distribution of particles along the wall is 
nonuniform. This time, the areas of higher particle concentration form larger fingers during particle transport; moreover, they absorb smaller fingers that form nearby. This process repeats for subsequent switches: each switch further reinforces the localization of particles in these larger fingers. Eventually, the system reaches a steady state in which the particles are in a well-defined pattern along the wall and form thick, periodicallyspaced fingers which resemble plumes during transport, as seen in the right panel of Figure 2. Because the pattern forms from the fastest-growing fingers, the characteristic wavelength of the periodic pattern matches $\lambda_{\mathrm{m}}$.

For $t_{\mathrm{s}}>2 \mathrm{~s}$, we determine $\lambda_{\mathrm{m}}$ by measuring the wavelength of the pattern, which we measure as the average distance between peaks in the particle distribution. The wavelength increases with increasing $t_{\mathrm{s}}$, as shown in Figure $4 \mathrm{c}$. Moreover, we find that $\lambda_{\mathrm{m}}$ scales with $t_{\mathrm{s}}^{1 / 2}$, as shown in Figure $4 \mathrm{~d}$; thus, it scales as a function of time in the same way as diffusion. By fitting the data, we find that $\lambda_{\mathrm{m}}$ grows as $22.4 t_{\mathrm{s}}{ }^{1 / 2}$; if we compare this coefficient with the measured ratio between $\lambda_{\mathrm{m}}$ and the cutoff wavelength, we obtain a prediction for the cutoff wavelength: $6 t_{\mathrm{s}}{ }^{1 / 2}$. This coefficient is consistent with our measurement of particle diffusion during the time between switches. Thus, our results are consistent with particle diffusion causing the largewavenumber cutoff in the growth rate curve.

The growth of fingers during particle transport can be compared to the Rayleigh-Taylor instability; similar behavior is also observed for a colloidal suspension sedimenting through a fluid. ${ }^{22}$ In our system, the positively charged particles are in a stable configuration when they are packed against the wall adjacent to the negative electrode by the applied field. When the direction of the field is switched, the system becomes unstable, as the particles must move through the particle-free fluid to reach their new stable state at the opposite wall. This is analogous to the sedimentation of colloids through a fluid, suggesting that the behavior of our system can be described by similar physics and also exhibits the Rayleigh-Taylor instability. At early times of the Rayleigh-Taylor instability, perturbations in the interface between the fluids are described by a linear stability criterium and are expected to grow exponentially in time; the linear stability regime is valid only when the amplitudes of the perturbations are much smaller than their wavelengths. However, in the time frame in which we observe our instability, the amplitudes of the fingers are large compared to their wavelengths; moreover, we measure the temporal growth of the modes to be linear, not exponential. Therefore, our instability has progressed beyond the linear to the nonlinear stability regime. Within the nonlinear regime of the Rayleigh-Taylor instability, the length scale of the fingers scales with the initial thickness of the unstable layer. ${ }^{23}$ Indeed, we find that, in our system, both the fastest-growing wavelength of the fingers and the thickness of the unstable particle layer increase with the time between consecutive switches. Thus, similar to the nonlinear Rayleigh-Taylor instability, an increase in the thickness of the particle layer corresponds to an increase in the fastest-growing wavelength of fingers during particle transport.

\section{CONCLUSIONS}

Here, we have used direct visualization of the dynamics of charged particles suspended in a nonpolar solvent to understand the origin of pattern formation in an applied electric field. We find that repeated switches in the direction of the applied field results in the localization of particles into a well-defined, periodic pattern. The pattern results from a fingering instability which occurs as the particles are transported across the channel. This fingering instability can be compared to the RayleighTaylor instability; however, determining the origin of the fielddriven fingering instability requires further study. For example, one possibility for future exploration is the Rayleigh-Bernard instability, which also leads to similar pattern-formation results. $^{24,25}$ Our findings provide a basis for explaining the formation of patterns in electric-field-driven particle suspensions and for developing methods to reduce unwanted behaviors in electrophoretic displays, which is essential for the continued improvement of display technology.

\section{ASSOCIATED CONTENT}

\section{Supporting Information}

Videos of particle transport across the microfluidic channel in response to a switch in the direction of the applied electric field; frame rate is $10 \mathrm{kHz}$. This material is available free of charge via the Internet at http://pubs.acs.org/.

\section{AUTHOR INFORMATION}

\section{Corresponding Author}

*E-mail: weitz@seas.harvard.edu.

Notes

The authors declare no competing financial interest.

\section{ACKNOWLEDGMENTS}

We thank Cabot Corporation for providing materials. We also thank Ian Morrison and Michael Brenner for helpful discussions and Esther Amstad for valuable feedback on the manuscript. This work was supported by the Harvard MRSEC (DMR-0820484) and the NSF (DMR-1310266). T.L. acknowledges support by the Department of Defense (DoD) through the National Defense \& Engineering Graduate Fellowship (NDSEG) Program and the National Science Foundation Graduate Research Fellowship.

\section{REFERENCES}

(1) Guo, Q.; Singh, V.; Behrens, S. H. Electric Charging in Nonpolar Liquids Because of Nonionizable Surfactants. Langmuir 2010, 26, 3203-3207.

(2) Morrison, I. D. Electrical Charges in Nonaqueous Media. Colloids Surf., A 1993, 71, 1-37.

(3) Hsu, M. F.; Dufresne, E. R.; Weitz, D. A. Charge Stabilization in Nonpolar Solvents. Langmuir 2005, 21, 4881-4887.

(4) Sainis, S. K.; Merrill, J. W.; Dufresne, E. R. Electrostatic Interactions of Colloidal Particles at Vanishing Ionic Strength. Langmuir 2008, 24, 13334-13337.

(5) Espinosa, C. E.; Guo, Q.; Singh, V.; Behrens, S. H. Particle Charging and Charge Screening in Nonpolar Dispersions with Nonionic Surfactants. Langmuir 2010, 26, 16941-16948.

(6) Guo, Q.; Lee, J.; Singh, V.; Behrens, S. H. Surfactant Mediated Charging of Polymer Particles in a Nonpolar Liquid. J. Colloid Interface Sci. 2013, 392, 83-89.

(7) Roberts, G. S.; Sanchez, R.; Kemp, R.; Wood, T.; Bartlett, P. Electrostatic Charging of Nonpolar Colloids by Reverse Micelles. Langmuir 2008, 24, 6530-6541.

(8) Smith, G. N.; Eastoe, J. Controlling Colloid Charge in Nonpolar Liquids with Surfactants. Phys. Chem. Chem. Phys. 2013, 15, 424-439.

(9) Hashmi, S. M.; Firoozabadi, A. Field- and ConcentrationDependence of Electrostatics in Non-Polar Colloidal Asphaltene Suspensions. Soft Matter 2012, 8, 1878-1883.

(10) Merrill, J. W.; Sainis, S. K.; Dufresne, E. R. Many-Body Electrostatic Forces Between Colloidal Particles at Vanishing Ionic Strength. Phys. Rev. Lett. 2009, 103, 138301. 
(11) Tettey, K. E.; Yee, M. Q.; Lee, D. Layer-by-Layer Assembly of Charged Particles in Nonpolar Media. Langmuir 2010, 26, 9974-9980.

(12) Novotny, V. Applications of Nonaqueous Colloids. Colloids Surf. 1987, 24, 361-375.

(13) Comiskey, B.; Albert, J. D.; Yoshizawa, H.; Jacobson, J. An Electrophoretic Ink for All-Printed Reflective Electronic Displays. Nature 1998, 394, 253-255.

(14) Murau, P.; Singer, B. The Understanding and Elimination of Some Suspension Instabilities in an Electrophoretic Display. J. Appl. Phys. 1978, 49, 4820-4829.

(15) Trau, M.; Sankaran, S.; Saville, D. A.; Aksay, I. A. Electric-FieldInduced Pattern Formation in Colloidal Dispersions. Nature 1995, 374, 437-439.

(16) Yeh, S.-R.; Seul, M.; Shraiman, B. I. Assembly of Ordered Colloidal Aggregates by Electric-Field-Induced Fluid Flow. Nature 1997, 386, 57-59.

(17) Trau, M.; Saville, D. A.; Aksay, I. A. Field-Induced Layering of Colloidal Crystals. Science 1996, 272, 706-709.

(18) Kim, J.; Garoff, S.; Anderson, J. L.; Schlangen, L. J. M. Movement of Colloidal Particles in Two-Dimensional Electric Fields. Langmuir 2005, 21, 10941-10947.

(19) Bartolo, D.; Degre, G.; Nghe, P.; Studer, V. Microfluidic Stickers. Lab Chip 2008, 8, 274-279.

(20) Lin, T.; Kodger, T. E.; Weitz, D. A. Transport of Charged Colloids in a Nonpolar Solvent. Soft Matter 2013, 9, 5173-5177.

(21) Sharp, D. An Overview of Rayleigh-Taylor Instability. Phys. D 1984, 12, 3-18.

(22) Wysocki, A.; Royall, C. P.; Winkler, R. G.; Gompper, G.; Tanaka, H.; van Blaaderen, A.; Lowen, H. Direct Observation of Hydrodynamic Instabilities in a Driven Non-Uniform Colloidal Disperson. Soft Matter 2009, 5, 1340-1344.

(23) Newhouse, L. A.; C. Pozrikidis, C. The Rayleigh-Taylor Instability of a Viscous Liquid Layer Resting on a Plane Wall. J. Fluid Mech. 1990, 217, 615-638.

(24) Turner, J. S. Double-diffusive Phenomena. Annu. Rev. Fluid Mech. 1974, 6, 37-54.

(25) Jerri, H. A.; Sheehan, W. P.; Snyder, C. E.; Velegol, D. Prolonging Density Gradient Stability. Langmuir 2009, 26, 47254731. 